\title{
Computational fluid dynamics simulations of single-phase flow in a filter- press flow reactor having a stack of three cells
}

Miguel A. Sandoval ${ }^{\mathrm{a}}$, Rosalba Fuentes ${ }^{\mathrm{a}}$, Frank C. Walsh ${ }^{\mathrm{b}}$, José L. Nava ${ }^{\mathrm{c}^{*}}$, Carlos Ponce de León $^{\mathrm{b}}$

${ }^{a}$ Universidad de Guanajuato, Departamento de Ingeniería Química, Norial Alta S/N, 36050

Guanajuato, Guanajuato, México. E-mail: ma.sandovallopez@ugto.mx; rosalba@ugto.mx

${ }^{\mathrm{b}}$ Electrochemical Engineering Laboratory, Energy Technology Research Group, Engineering Sciences, University of Southampton, Highfield, Southampton, SO17 1BJ, United Kingdom. E-mail: F.C.Walsh@soton.ac.uk; capla@soton.ac.uk

${ }^{\text {c } U n i v e r s i d a d ~ d e ~ G u a n a j u a t o, ~ D e p a r t a m e n t o ~ d e ~ I n g e n i e r i ́ a ~ G e o m a ́ t i c a ~ e ~ H i d r a ́ u l i c a, ~ A v . ~}$ Juárez 77, Zona Centro, 36000 Guanajuato, Guanajuato, México. E-mail: jlnm@ugto.mx

*Corresponding author: jlnm@ugto.mx

Tel: + 52-4731020100 ext. 2289; fax: + 52-4731020100 ext. 2209. 


\begin{abstract}
Computational fluid dynamics (CFD) simulations were carried out for single-phase flow in a pre-pilot filter press flow reactor with a stack of three cells. Velocity profiles and streamlines were obtained by solving the Reynolds-Averaged Navier-Stokes (RANS) equations with a standard $k-\varepsilon$ turbulence model. The flow behaviour shows the appearance of jet flow at the entrance to each cell. At lengths from 12 to $15 \mathrm{~cm}$ along the cells channels, a plug flow pattern is developed at all mean linear flow rates studied here, $1.2 \leq u$ $\leq 2.1 \mathrm{~cm} \mathrm{~s}^{-1}$. The magnitude of the velocity profiles in each cell was different, due to the turbulence generated by the change of flow direction in the last fluid manifold. Residence time distribution (RTD) simulations indicated that the fluid behaviour tends towards a continuous mixing flow pattern, owing to flow at the output of each cell across the upper cell link pipe, which acts as a mixer. Close agreement between simulations and experimental RTD was obtained.
\end{abstract}

Keywords: Computational Fluid Dynamics (CFD), Filter press flow reactor, Multielectrode stack, Residence time distribution (RTD), Pressure drop. 


\section{Introduction}

Filter-press electrochemical reactors fitted with parallel plate electrodes are frequently used in industrial practice and as multipurpose flow cells employed in a diverse range of electrochemical processes [1]. The design of a filter-press electrolyser must consider appropriate characterization of the reaction environment to provide uniform current and potential distributions as well as effective heat and flow dispersion [2, 3]. In a previous paper from our laboratories, the reaction environment was characterised in a well-known filter press electrolyser in pre-pilot scale (the FM01-LC) [2], which has been widely studied from the theoretical and experimental point of view, demonstrating the effectiveness of the reactor for a diverse range of applications that include organic and inorganic electrosynthesis, metal ion removal, energy storage, environmental remediation and drinking water treatment [1].

During the characterization of the reaction environment, the hydrodynamics play an important role, because the flow environment around the electrodes determines the magnitude and uniformity of mass transport and consequently the current distribution, current efficiency and energy consumption during electrolysis. The accurate study of flow patterns is essential to design the components of the flow cells, such as flow distributors, net-like spacers employed as turbulence promoters and cell dimensions (i.e., height, width and length) to guarantee homogeneous velocity fields while avoiding undesirable flow features, such as stagnant zones, back-mixing and electrolyte recirculation. 
The modelling and simulation of fluid hydrodynamics in filter-press electrolysers has recently been performed by computational fluid dynamics (CFD) techniques using commercial and open software, enabling a comprehensive and detailed description of the local hydrodynamic within the flow cells [4-16]. Different CFD methods have been used to characterize the liquid flow pattern, as is the case of the residence time distribution (RTD). The Navier-Stokes (NS) equations coupled with the diffusion-convection equation, and Reynolds averaged Navier Stokes (RANS) equations coupled with averaged convection equation, have been solved for laminar and turbulence regimes, respectively [8-9, 11]. It is important to highlight that theoretical CFD studies in multi-stack filter press electrolysers have rarely been published in the literature and there are no studies on residence time distribution in multi-electrode stacks. Walsh and coworkers have reported experimental results of RTD in an industrial filter-press redox flow reactor containing a bipolar stack of five cells used for the $\mathrm{Na}_{2} \mathrm{~S} / \mathrm{NaBr}$ system [3]; each bipolar cell consisted of two electrodes with a projected area of $0.72 \mathrm{~m}^{2}(66.8 \mathrm{~cm} \times 108.0 \mathrm{~cm})$ separated by $0.2 \mathrm{~cm}$ interelectrode gap. Experimental RTD data, achieved by an electrolytic conductivity method, revealed the appearance of slow and fast phases, with the slow phase disappearing at the highest flow rates. It is important to emphasize that in experimental RTD tests, the tracer is normally measured at the exit of the cell in a 'black box' approach; flow within the entrance flow distributors, net-like spacer employed as turbulence promoter and cell corners is hidden. CFD simulations coupled with experimental RTD allow the local causes of flow deviations to be identified and support the design of some components of the multi-electrode stack to prevent undesirable flow patterns [4]. 
In filter-press flow reactors using a multi-electrode stack, the cross-sectional area changes of the flow, induce velocity field variations in a random fashion, which generates jet flows, vortex and rotational flow structures, so-called turbulent eddies [17]. In such a complex geometry, turbulence models can be used to simulate realistic flow patterns [14]. In this paper, we solve the RANS equations with the $\mathrm{k}-\varepsilon$ turbulence model for the CFD simulations.

The objective of the present paper is to simulate the single-phase flow in a filter press flow reactor with a stack of three cells. The reactor employed is the FM01-LC electrolyser, which has been recently reviewed $[1,2]$. CFD simulations of hydrodynamics were obtained solving the RANS equations with the $\mathrm{k}-\varepsilon$ turbulence model. RTD simulations were obtained solving the averaged diffusion-convection equation. Theoretical RTD values were validated by experimental data. Pressure drop measurements were also examined since such measurements are relevant to electrolyte pumping requirements and costs.

\section{Description of the reactor}

The electrochemical reactor is shown in Figure 1 and consists of a four-electrode stack of three undivided cells. The electrodes were electrically connected in a monopolar configuration. The characteristics of the filter press reactor are given in Table 1 and detailed description can be found elsewhere $[1,18]$. Four aluminum plates (99.7\% purity) were fitted between spacers to form a stack of three cells; the electrolyte flowed upwards past the vertical electrodes within three parallel, rectangular flow channels. The net spacing between the aluminum electrodes was $0.6 \mathrm{~cm}$, a distance being controlled by polypropylene 
separators acting as fluid distributors. The fluid was fed through a $1.3 \mathrm{~cm}$ diameter circular tube. The fluid distributor contains five inlet manifolds (Fig. 2b) which took the electrolyte across the inter-electrode space, and at the top, five outlet manifolds take the electrolyte out through a $1.3 \mathrm{~cm}$ diameter circular tube. At the entrance of the cell, a $1.3 \mathrm{~cm}$ diameter cell link pipe, between polypropylene separators and electrodes (Fig. 2) acted on the front face of the first fluid distributor ending at the back of the third cell. The total length of the bottom link pipe was $7 \mathrm{~cm}$, while the upper link pipe was $10 \mathrm{~cm}$ long.

\section{Formulation of the numerical simulation}

The volumetric flow rates studied were $8.3,10,11.7,13.3$ and $15 \mathrm{~cm}^{3} \mathrm{~s}^{-1}$ giving mean linear flow rates $(U)$ of $1.2,1.4,1.6,1.8$, and $2.1 \mathrm{~cm} \mathrm{~s}^{-1}$ obtaining Reynolds numbers $\left(R e=U d_{h} / v\right.$ ) ranging from 125 to 219 , where $U$ is the mean linear flow rate (evaluated using the cross sectional area of the three parallelepiped flow channels), $d_{h}$ is the hydraulic diameter, and $v$ is the kinematic viscosity. In multi-sided geometries, such as those present in the reactor studied here, turbulence models can be used to simulate realistic flow patterns. These flow patterns can be obtained by solving the RANS and the averaged diffusion-convection equations. For the solution of the RANS equations, a standard $k-\varepsilon$ turbulent model was used [9, 19].

\subsection{Turbulent flow}

Under turbulent flow conditions, the equations for an incompressible fluid can be stated as follows. The RANS and the continuity equations are: 
$\rho(\boldsymbol{u} \cdot \nabla) \boldsymbol{u}=-\nabla P+\nabla \cdot\left(\left(\mu+\mu_{T}\right)\left(\nabla \cdot \boldsymbol{u}+(\nabla \cdot \boldsymbol{u})^{T}\right)\right)$

$\nabla \cdot(\rho \boldsymbol{u})=0$

where $\boldsymbol{u}$ is the mean-averaged velocity vector, $P$ the pressure, $\mu$ the dynamic viscosity, $\rho$ the fluid density. The notation $O^{T}$ indicates the transpose of $\nabla \cdot u$, and it should not be confused with any turbulent suffix. The so-called Reynolds stresses can be expressed in terms of a turbulent viscosity $\mu_{T}$, according to the standard $k-\varepsilon$ turbulence model:

$\mu_{T}=\rho C_{\mu} \frac{k^{2}}{\varepsilon}$

$\rho(\boldsymbol{u} \cdot \nabla) k=\nabla \cdot\left(\left(\mu+\frac{\mu_{T}}{\sigma_{k}}\right) \nabla k\right)+P_{k}-\rho \varepsilon$

$\rho \boldsymbol{u} \cdot \nabla \varepsilon=\nabla \cdot\left(\left(\mu+\frac{\mu_{T}}{\sigma_{\varepsilon}}\right) \nabla \varepsilon\right)+C_{e 1} \frac{\varepsilon}{\kappa} P_{k}-C_{e 2} \rho \frac{\varepsilon^{2}}{k}$

where $k$ is the turbulent kinetic energy, $\varepsilon$ is the turbulent energy dissipation rate, $P_{k}$ is the energy production term $\left(P_{k}=\mu_{T}\left[\nabla \boldsymbol{u}:\left(\nabla \cdot \boldsymbol{u}+(\nabla \cdot \boldsymbol{u})^{T}\right]\right)\right.$, and $C_{\mu}(0.09), C_{e l}(1.44), C_{e 2}(1.92)$, $\sigma_{k}(1), \sigma_{\varepsilon}(1.3)$ are dimensionless constant values that are obtained by data fitting over a wide range of turbulent flows $[17,20]$.

This model is applicable at high Reynolds numbers; wall functions are typically used to solve such problems. The functions involved are based on a universal velocity distribution which, in a turbulent layer, can be described by: 
$u^{+}=2.5 \ln y^{+}+5.5$

Here, $u^{+}$is the normalized velocity component inside the logarithmic boundary layer, and $y^{+}$is the dimensionless distance from the wall, $y^{+}=\rho u_{\tau} y / \mu$, where $u_{\tau}$ is the friction velocity, $u_{\tau}=C_{\mu}^{1 / 4} \sqrt{k}$ and $y$ is the distance from the wall $[17,20]$.

The boundary conditions to solve Eqs. (1) to (5) are:

- That the normal inflow velocity at the electrolyte inlet should be: $\boldsymbol{u}=-U_{0} \cdot \boldsymbol{n}$, where $U_{0}$ is the average velocity fluid at the entrance and $\boldsymbol{n}$ is the unit normal vector; in this work the approximation for the inlet values of $k_{0}$ and $\varepsilon_{0}$ were obtained from the turbulent intensity $I_{T}$, and the turbulent length scale $L_{T}$, by means of the following simple assumed forms: $k_{o}=3 / 2\left(U_{o} I_{T}\right)^{2}$ and $\varepsilon_{0}=C_{\mu}^{3 / 4} k^{3 / 2} / L_{T}$ [17]. The turbulent intensity for fully turbulent flows has dimensionless values between 0.05 and 0.1 . The turbulent length scale can be determined in pipes as a function of the radius by means of $L_{T}=0.07 r$, where $r$ is the inlet radius of $0.65 \mathrm{~cm}$. In this work, $I_{T}$ and $L_{T}$, were fixed at 0.05 and $0.0455 \mathrm{~cm}$, respectively.

- That the normal stress is equal to the pressure at the outlet, $\left[-P+\left(\mu+\mu_{T}\right)(\nabla \boldsymbol{u}+\right.$ $\left.\left.(\nabla \boldsymbol{u})^{T}\right) \cdot \boldsymbol{n}\right]=-P_{0} \cdot \boldsymbol{n}$ where $P_{0}$ is the pressure at the electrolyte exit. This last equation expresses that the turbulent characteristic of whatever is outside the computational domain is guided by the flow inside the computational domain [21]. Such an assumption is physically reasonable as long as relatively small amounts of fluid enter the system. Moreover, at the electrolyte outlet, $\nabla \varepsilon \cdot n=0$ and $\nabla k \cdot n=0$. 
- That the velocity, $u^{+}$for all other boundaries is given by equation (6) at a distance $y^{+}$ from a solid surface.

After verifying the solution at different values of $y^{+}$and step sizes, the value of $y^{+}$was fixed at 11.1. This value is in the fully turbulent region $\left(5<y^{+}<30\right)$, where the turbulent stresses and fluxes are more important [22].

\subsection{Residence time distribution}

The time-dependent behavior of a tracer inside the empty cell (in laminar flow) can be described by the general form of the diffusion-convection equation:

$\frac{\partial C}{\partial t}=-\boldsymbol{u} \cdot \nabla C+\nabla \cdot\left(D+D_{T}\right) \nabla C$

where $C$ is the averaged concentration of the tracer, $t$ is the time, $D$ is the diffusion coefficient, $\boldsymbol{u}$ is the velocity vector obtained by the solution of Equations (1) and (2) and $D_{i, T}$ is the eddy diffusivity or turbulent diffusivity. Eddy diffusivity can be determined from the turbulent Schmidt number $\left(S c_{T}=\mu_{T} /\left(\rho D_{T}\right)\right)$ described by the following KaysCrawford model [23]:

$S c_{T}=\left(\frac{1}{2 S c_{T \infty}}+\frac{0.3}{\sqrt{S c_{T} \infty}} \frac{\mu_{T}}{\rho D_{i}}-\left(0.3 \frac{\mu_{T}}{\rho D_{i}}\right)\left(1-\exp \left(-\frac{\rho D_{i}}{0.3 \mu_{T} \sqrt{S c_{T \infty}}}\right)\right)\right)^{-1}$ 
where $S c_{T \infty}=0.85$.

Considering perfect mixing conditions before the inlet and after the outlet of the reactor, the boundary and the initial conditions can be established as follows:

- The tracer concentration is zero, $C=0$, before the tracer injection in the reactor $(t=$ $0)$.

To simulate the tracer pulse injection, we employed a Gaussian pulse function:

$$
y(t)=\frac{1}{\sigma \sqrt{2 \pi}} e^{\frac{-(t-t=0)^{2}}{2 \sigma^{2}}}
$$

At the inlet, $C=C_{0} y(t) / y(0)$. Here, $C_{0}$ is the initial tracer concentration $\left(\mathrm{Cu}^{2+} 0.005 \mathrm{~mol}\right.$ $\mathrm{cm}^{-3}$ ). To simulate the tracer injection in the time interval, a Gaussian pulse function was varied from 3 to $4 \mathrm{~s}$ using a standard deviation, $\sigma$ from 1.5 to 2.7 .

- At the electrolyte outlet, $\boldsymbol{n} \cdot\left(-\left(D+D_{T}\right) \nabla C+\boldsymbol{u} \cdot \nabla C\right)=0$.

- For all other boundaries, $-\boldsymbol{n} \cdot N=0$, where $N$ is the flux of tracer.

The simulation of the normalized RTD curve, $E(t)$, which describes the tracer distribution in certain periods of time for the stream of fluid leaving the reactor, can be assessed according to [24]: 
$E(t)=\frac{C(t)}{\int_{0}^{\infty} C(t) d t}$

where $C(t)$ is the time-dependent concentration response. Simulations of RTD curves in the 3 cell modular stack were performed taking $C(t)$ from Eq. (7).

A dimensionless $E(\theta)$ function can be defined as:

$E(\theta)=\tau E(t)$

and can be plotted as function of the dimensionless time $\theta(=t / \tau)$, where $\tau$ is the spatial residence time $(=V / Q), V$ is the electrolyte volume inside the FM01-LC, and $Q$ is the volumetric flow rate. For the numerical simulation, $V=130 \mathrm{~cm}^{3}$.

\subsection{Simulation}

Transport Eqs. (1-5) and (7) were solved numerically in 3D by finite element method using the software COMSOL Multiphysics ${ }^{\circledR} 5.1$ on a computer with two Intel ${ }^{\circledR}$ Xeon ${ }^{\text {TM }} 2.30$ GHz processor, 96 GB of RAM, and 64-bit operating system.

Fig. 2 shows the computational domain, which considers 183,430 tetrahedral mesh elements. The wall roughness was assumed to have a negligible effect. The simulation run times were typically from 25 to 50 minutes depending on the flow rate. The typical solution around these mesh elements was unchanged. The solver employed was iterative, GMRES, 
and a relative tolerance of accuracy of the CFD simulations considered a convergence criterion below $1 \times 10^{-5}$.

\section{Experimental details}

\subsection{Equipment}

A potentiostat/galvanostat model SP-150 Biologic ${ }^{\mathrm{TM}}$ with EC-Lab ${ }^{\mathrm{TM}}$ software was used for experimental RTD determinations. Section 2 described the details of the filter press reactor used to perform the experimental determinations of RTD. A magnetically coupled, 1/10 hp pump (Little Giant model 4-MD-HC) was used to supply electrolyte flow. A $0.1 \mathrm{~L} \mathrm{~min}^{-1}$ to $1 \mathrm{~L} \mathrm{~min}^{-1}$ White industries flowmeter model F44250 was used to measure the volumetric flow.

\subsection{Residence time distributions experiments}

In order to determine the mixing flow pattern in the liquid phase, the stimulus-response technique was employed. Figure 3 shows a schematic diagram of the system used for the experimental RTD and pressure drop measurements. Tubes, valves and accessories were made of PVC with 0.5 inch diameter. $0.5 \mathrm{M}$ copper sulfate $(1 \mathrm{~mL})$ was employed as a tracer injected by syringe at a $1.5 \mathrm{~cm}$ distance before the reactor inlet. At the reactor outlet, the $\mathrm{Cu}^{2+}$ ion concentration was measured online by a typical, two-electrode cell arrangement using copper wire electrodes; the cupric ions were quantified by the typical transient current at a holding cell potential of $-0.9 \mathrm{~V}$ and the current response was measured with a potentiostat-galvanostat. It is important to mention that at a cell potential of $-0.9 \mathrm{~V}$, a convective-diffusion, mass transport limiting current for copper deposition controls the 
cathodic process, ensuring that the response only depends on the cupric ion concentration [19]. This quantification method was sufficiently fast and sensitive to capture the rapid concentration changes at the reactor outlet as seen in the RTD curves shown below.

For the pressure drop measurements a $60 \mathrm{~cm}$ tall, vertical glass, U-tube, water-filled manometer was employed, using water/gel mixture as the fluid. The manometer was connected by silicone tubing to tees located as close as possible to the inlet and outlet of the reactor. The RTD and pressure drops measurements were performed at different volumetric flow rates from 8.3 to $15 \mathrm{~cm}^{3} \mathrm{~s}^{-1}$, corresponding to a mean linear flow velocity range from 1.2 to $2.1 \mathrm{~cm} \mathrm{~s}^{-1}$, evaluated using the cross sectional area of the three parallelepiped flow channels.

\section{Results and discussion}

\subsection{CFD simulations}

Fig. 4 shows the velocity magnitude field inside the filter-press with a four-electrode stack of three cells at a mean linear flow velocity $U=1.8 \mathrm{~cm} \mathrm{~s}^{-1}$. The velocity field shows the common inlet manifold effects (bottom zone) associated with the variations in the cross sectional area. Afterwards, the velocity tends to homogenize in the three parallelepiped zones; at the exit (upper zone) the velocity increases. Regarding the position of the cells, the higher velocities increased in cell 3 , as seen from the lower flow contours in the inset. In the upper cell link pipe, the fluid contours developed a spiral flow pattern achieving maximum values close to the centre. This indicates that the upper cell link pipe acts as a 
fluid mixer. A similar situation persists in the inlet of the cell (see bottom flow contours). In order to determine the hydrodynamic behavior of Figure 4, velocity profiles along the cell (x-coordinate) were constructed.

Fig. 5 shows the velocity profiles in the cell width (in the $y$-coordinate) taken at different heights of $0.3,3,8$ and $15 \mathrm{~cm}$, in the $x$-coordinate, and at depth of $0.1 \mathrm{~cm}$ (in the $z$-coordinate) in each cell. Close to the entrance, at $x=0.3 \mathrm{~cm}$, five maximum velocities (jet flows) appeared originated by the inlet manifolds, reaching velocities from 0.17 to $4.18 \mathrm{~cm}$ $\mathrm{s}^{-1}$, highlighting that the velocity magnitude increases in the following order cell $1<$ cell 2 $<$ cell 3. The latter originates from turbulence generated by the change of flow direction in the third cell, see the bottom flow contour in Fig. 4. At a $3 \mathrm{~cm}$ height, the velocity profiles are attenuated for the three cells and only two velocity maximums appeared; then, at an 8 $\mathrm{cm}$ height, an almost flat profile is achieved and at a $15 \mathrm{~cm}$ height, a plug flow pattern was fully developed. Velocity profiles constructed at $8<x<15 \mathrm{~cm}$ (not shown) confirmed that the full plug flow pattern is reached between 12 and $15 \mathrm{~cm}$ height, for the flow velocities between 1.2-2.1 $\mathrm{cm} \mathrm{s}^{-1}$ (Reynolds ranged between 125-219). According to Vazquez et al. [6], the fully developed plug flow can be achieved at heights from 4 to $15 \mathrm{~cm}$ (in the $x$ coordinate), at Reynolds from 150 to 550; it is important to realise that Vazquez et al. [6] used the FM01-LC with a single cell. The flow pattern they experienced is different to that obtained here owing to fluid flow variations being determined by the geometry of the multicell arrangement.

In order to perform a more complete flow pattern characterization inside the multi-electrode 
stack reactor, RTD studies were used to validate the CFD simulations. Fig. 6 shows the comparison between the experimental and theoretical RTD curves at different mean linear flow rates. The simulated RTD curves were in good agreement with experimental ones. It can be observed that as the fluid flow rate increases, the dimensionless curves, $E(\theta)-\theta$, become narrower, and the maximum values of $E(\theta)$ become larger. Moreover, the RTD curves tend to the left with flow rate and the maximum of the RTD curve appeared at $\theta<1$. It is important to highlight that the tail decreases as the volumetric flow rate increases. The flow patterns obtained here indicate a better trend towards a continuous mixing flow pattern. All curves show that some elements of the tracer leave the reactor earlier than the average residence time $(\theta<1)$. According to the velocity profiles shown in Fig. 5 we expected a quasi-plug flow pattern in the RTD curves (i.e., obtaining the maximum of the RTD curves at $\theta=1$ ), although, the upper cell link pipe acted as a fluid mixer, modifying the shape of the RTD curves. This latter did not happen in the FM01-LC with a single cell $[9,25-26]$.

Fig. 7 shows the comparison of the theoretical tracer concentration distribution along the first, second and third cells respectively, at an inflow volumetric flow rate of $13.3 \mathrm{~cm}^{3} \mathrm{~s}^{-1}$, and at different times. At time of $1 \mathrm{~s}$ and $3 \mathrm{~s}$, we can observe that the tracer concentration increases in the following order: cell $1>$ cell $2>$ cell 3 , indicating that the fluid elements come earlier into the first cell, next the second cell, then the third cell. Afterwards, at time of $9 \mathrm{~s}$ the tracer concentration follows the order: cell $1<$ cell $2<$ cell 3 , because the fluid elements in the first cell leave the reactor before the second and third cell; this latter is more evident at time of $19 \mathrm{~s}$, where the tracer concentration in the first cell is practically zero. 
Similar tracer analysis performed at inflow rates of $8.3,10,11.7$, and $15 \mathrm{~cm}^{3} \mathrm{~s}^{-1}$ (not shown herein) developed similar patterns to that obtained at $13.3 \mathrm{~cm}^{3} \mathrm{~s}^{-1}$.

Fig. 8 (a)-(b) shows the streamlines for lateral view and the back view of the third cell, respectively, at $Q=13.3 \mathrm{~cm}^{3} \mathrm{~s}^{-1}$. The lateral view shows the homogeneous flow path highlighting that in the upper cell link pipe the streamlines are more concentrated, although, this flow path did not show any back mixing or recirculation zones. The streamlines at the back view of the third cell, Fig. 8(b), also confirms the absence of back mixing or recirculation zones. The fluid elements in the first and second cells leave the reactor before the third cell (Figure 7). This latter in connection with the mixing in the upper cell link pipe explains the presence of the maximum RTD curve at $\theta<1$, in Fig. 6. Finally, the homogeneous streamlines path (Fig. 8) in connection with tail depletion as the volumetric flow rate increases (Fig. 6) provides evidence that the tail in the RTD curves results from loss of kinetic energy in the many-sided geometry and by the turbulent energy dissipation rate. This is supported by the velocity diminution at each cell at $15 \mathrm{~cm}$ distance from the fluid entrance as shown in Fig. 5 (close to the cell exit), where the local flow velocities were lower than the mean linear flow velocity $U=1.8 \mathrm{~cm} \mathrm{~s}^{-1}$. The velocity is also related to the pressure drop in the multi-electrode stack, as discussed below.

CFD simulations (not shown) were performed using other flow model, solving the RANS equations for low Reynolds number (RANS-LRN) and the averaged convection-diffusion equation for RTD studies [9], highlighting that after several attempts, CFD simulations did not match the experimental RTD curves. This confirmed that the model proposed here to 
simulate the non-ideal flow in filter press reactor with multi-electrode stack is appropriate.

\subsection{Pressure drop and flow}

Pressure drop analysis is important in order to calculate the energy necessary to realize a determined flow velocity in filter press reactors. Experimental results have suggested that the pressure drop $(\Delta P)$ is a logarithmic function of the flow velocity, expressed in terms of the Reynolds number. Several literature reports have focused on the characterization of the pressure drop across the FM01-LC reactor as a function of Reynolds via an empirical power law:

$$
\Delta P=a \mathrm{Re}^{b}
$$

Fig. 9 shows the logarithmic plot of the pressure drop as a function of Reynolds number in the multi-electrode stack and the reported in the literature for empty single cell and filled single cell with turbulence promoter [27]. From the analysis of this Figure we can observed that the pressure drop increases with linear fluid velocity. Table 2 summarizes the experimental parameters reported in the literature at different flow configurations in comparison to those obtained in this work. In the case of the stack with three cells, the $a$ value (associated with the geometry) decreases in comparison with the value obtained for a single cell and a single cell with a turbulence promoter, as shown in Table 2 . Whereas the $b$ index (associated with hydrodynamics) increases in comparison to one empty cell, and the cell with turbulence promoter. The pressure drop increase in the multi-electrode stack is expected because the energy required to impose flow fluid becomes greater in the stack 
with three cells. From the engineering standpoint, the stack of three cells demands a higher pumping power.

\section{Conclusions}

This work has presented a theoretical study for the hydrodynamics in a pre-pilot filter press flow electrolyser with a stack of three cells. We believe that this is the first time that a CFD simulation has successfully considered the presence of three cells in a multi-electrode stack. CFD simulations have shown the appearance of 5 jets flow at the entrances of each cell, the plug flow being fully developed at lengths between 12 and $15 \mathrm{~cm}$. It is important to highlight that velocity magnitude increases in the following order cell $1<$ cell $2<$ cell 3 . The latter is caused by the turbulence due to the change of flow direction in the third cell.

According to CFD simulations reported in the literature for the single cell FM01-LC reactor, quasi-plug flow is achieved at heights from $4.0 \mathrm{~cm}$ to $15.0 \mathrm{~cm}$. This latter pattern is different to that obtained here owing to the fluid flow variations being determined by larger cross-sectional area of the multi-electrode stack compared to the single cell reported in the literature. The simulated RTD curves were in good agreement with experimental ones. However, the maximum values of RTD curves appeared before the unity with respect to the dimensionless time, contrary to that obtained in the single cell FM01-LC (where the maximum appear close to unity regarding the dimensionless time). The flow patterns obtained here indicate a trend towards a continuous mixing flow pattern. This latter pattern is determined by the upper cell link pipe, which acts as a fluid mixer. The numerical simulation model proposed here could be used to predict mass transport simulations 
allowing tertiary current and potential distributions across the flow cells in multi-electrode stack to be simulated.

\section{Acknowledgments}

M. Sandoval is grateful to CONACYT for financial support under the 386022 scholarship. J.L. Nava acknowledges Universidad de Guanajuato for financial support through the project No. $886 / 2016$. 


\section{References}

[1] F.F. Rivera, C. Ponce de León, J.L. Nava, F.C. Walsh, The filter-press FM01-LC laboratory flow reactor and its applications, Electrochim. Acta 163 (2015) 338-354.

[2] F.F. Rivera, C. Ponce de León, F.C. Walsh, J.L. Nava, The reaction environment in a filter-press laboratory reactor: the FM01-LC flow cell, Electrochim. Acta 161 (2015) 436452.

[3] C. Ponce de León, G.W. Reade, I. Whyte, S.E. Male, F.C. Walsh, Characterization of the reaction environment in a filter-press redox flow reactor, Electrochim. Acta 52 (2007) $5815-5823$

[4] M.J. Nieves-Remacha, A.A. Kulkarni, K.F. Jensen, Open FOAM Computational fluid dynamic simulations of single-phase flows in an advanced-flow reactor, Ind. Eng. Chem. Res. 54 (2015) 7543-7553.

[5] E. P. Rivero, F. F. Rivera, M. R. Cruz-Díaz, E. Mayen, I. González, Numerical simulation of mass transport in a filter press type electrochemical reactor FM01-LC: Comparison of predicted and experimental mass transfer coefficient, Chem. Eng. Res. Des. 90 (2012) 1969-1978.

[6] L. Vázquez, A. Alvarez-Gallegos, F. Z. Sierra, C. Ponce de León, F. C. Walsh, Simulation of velocity profiles in a laboratory electrolyser using computational fluid dynamics, Electrochim. Acta 55 (2010) 3437-3445. 
[7] L. Vázquez, A. Alvarez-Gallegos, F.Z. Sierra, C. Ponce de León, F.C. Walsh, Prediction of mass transport profiles in a laboratory filter-press electrolyser by computational fluid dynamics modelling, Electrochim. Acta 55 (2010) 3446-3453.

[8] Á. Frias-Ferrer, I. Tudela, O. Louisnard, V. Sáez, M. D. Esclapez, M.I. Díez-García, P. Bonete, J. González-García, Optimized design of an electrochemical filter-press reactor using CFD methods, Chem. Eng. J. 169 (2011) 270-281.

[9] M.R. Cruz-Díaz, E.P. Rivero, F.. Almazán-Ruiz, Á. Torres-Mendoza, I. González, Design of a new FM01-LC reactor in parallel plate configuration using numerical simulation and experimental validation with residence time distribution (RTD), Chem. Eng. Process. 85 (2014) 145-154.

[10] G. Nelissen, B. Van De Bossche, J. Deconinck, A. Van Theemsche, C. Dan, Laminar and turbulent mass transfer simulations in a parallel plate reactor, J. Appl. Electrochem. (2003) 863-873.

[11] C. Bengoa, A. Montillet, P. Legentilhomme, J. Legrand, Characterization and modeling of the hydrodynamic behavior in the filter-press-type FM01-LC electrochemical cell by direct flow visualization and residence time distribution, Ind. Eng. Chem. Res. 39 (2000) 2199-2206. 
[12] M. Cruz-Díaz, F.F. Rivera, E.P. Rivero, I. González, The FM01-LC reactor modeling using axial dispersion model with a reaction term coupled with a continuous stirred tank (CST), Electrochim. Acta, 63 (2012) 47- 54.

[13] J.A Delgadillo, R. Enciso, C. Ojeda, I. Rodríguez, A comparative study of the turbulence-closure model for a FM01-LC electrochemical reactor, Int. J. Electrochem. Sci., 7 (2012) $2065-2076$.

[14] T. Pérez, C. Ponce de León, F.C. Walsh, J.L. Nava, Simulation of current distribution along a planar electrode under turbulent flow conditions in a laboratory filter-press flow cell, Electrochim. Acta 154 (2015) 352-360.

[15] J.L.C. Santos, V. Geraldes. S. Velizarov, J.G. Crespo, Characterization of fluid dynamics and mass-transfer in an electrochemical oxidation cell by experimental and CFD studies, Chem. Eng. J. 157 (2010) 379-392.

[16] U.M. López-García, P.E. Hidalgo, J. C. Olvera, F. Castañeda, H. Ruiz, G. Orozco, The hydrodynamic behavior of a parallel-plate electrochemical reactor, Fuel 110 (2013) 162170.

[17] H.K. Versteeg, W. Malalasekera, An Introduction to Computational Fluid Dynamics: The Finite Volume Method, Second ed., Prentice Hall, London, 1995. 
[18] O.J. Flores, J.L. Nava, G. Carreño, Arsenic removal from groundwater by electrocoagulation process in a filter-press type FM01-LC reactor, Int. J. Electrochem. Sci. 9 (2014) 6658-6667.

[19] M. Rosales, T. Pérez, J.L. Nava, Computational fluid dynamic simulations of turbulent flow in a rotating cylinder electrode reactor in continuous mode of operation, Electrochim. Acta 194 (2016) 338-345.

[20] P.S. Bernard, J.M. Wallace, Turbulent Flow: Analysis, Measurement and Prediction, first ed., John Wiley \& Sons, New Jersey, 2002.

[21] D.C. Wilcox, Turbulence Modeling for CFD, DCW Industries Inc, California, 1998.

[22] H. Schlichting, Boundary-Layer Theory, 7th ed., MC Graw-Hill, New York, 1979.

[23] B. Weigand, J.R. Ferguson, M.E. Crawford, An extended Kays and Crawford turbulent Prandtl number model, Int. J. Heat Mass Tran. 40(1997) 4191-4196.

[24] H.S. Fogler, Elements of Chemical Reaction Engineering, fourth, Prentice Hall, New Jersey, 2005. 
[25] P. Trinidad, F.C. Walsh, Hydrodynamic behaviour of the FM01-LC reactor, Electrochim. Acta 41 (1996) 493-502.

[26] P. Trinidad, C. Ponce de León, F.C. Walsh, The application of flow dispersion models to the FM01-LC laboratory filter-press reactor, Electrochim. Acta 52 (2006) 604-613.

[27] C.J. Brown, D. Pletcher, F.C. Walsh, J.K. Hammond, D. Robinson, Studies of spaceaverage mass transport in the FM01-LC laboratory electrolyser. J. Appl. Electrochem. 23 (1993) $38-43$. 
Table 1. Reactor parameters and electrolyte properties for the four-electrode stack of three undivided cells.

\begin{tabular}{lc}
\hline Volume $^{*}, V / \mathrm{cm}^{3}$ & 130 \\
Cell width, $B / \mathrm{cm}$ & 4.0 \\
Cell thickness, $S / \mathrm{cm}$ & 0.6 \\
Cell length, $L / \mathrm{cm}$ & 16.0 \\
Number of cells & 3 \\
Total length, $L_{T} / \mathrm{cm}$ & 48 \\
Equivalent diameter of flow cell thickness, $d_{h}=2 \mathrm{BS} / \mathrm{B}+\mathrm{S} / \mathrm{cm}$ & 1.04 \\
Anode area in each cell in contact with solution $/ \mathrm{cm}^{2}$ & 64 \\
Cathode area in each cell in contact with solution $/ \mathrm{cm}^{2}$ & 64 \\
Kinematic viscosity, $v / \mathrm{cm}^{2} \mathrm{~s}^{-1}$ & 0.01 \\
\hline
\end{tabular}

*It includes the volume of the cell link pipes plus fluid manifolds.

Table 2. Experimental values of pressure drop $\left(\Delta P=a R e^{b}\right)$ over the FM01-LC reactor.

\begin{tabular}{llll}
\hline Configuration & $a / \times 10^{2} \mathrm{~Pa}$ & $b$ & Reference \\
\hline Stack of three undivided cells & 0.028 & 2.88 & This work \\
Empty single cell & 0.69 & 1.39 & {$[27]$} \\
Filled single cell with PTFE turbulence promoter type D & 1.69 & 1.54 & {$[27]$}
\end{tabular}




\section{Figure Captions}

Fig. 1. The FM01-LC electrochemical reactor with a four-electrode stack of three undivided cells. The electrodes are switched in monopole configuration.

Fig. 2. (a) Computational domain used in the numerical simulation. (b) Schematic in the $x-y$ plane of a single cell. The interelectrode space is a rectangular flow channel $4 \mathrm{~cm}$ wide, $16 \mathrm{~cm}$ long and $0.6 \mathrm{~cm}$ deep. Volume of the domain, $V=130 \mathrm{~cm}^{3}$.

Fig. 3. The flow system used for residence time distribution experiments.

Fig. 4. Velocity magnitude field distributions inside the reactor for the entire domain, for a mean linear flow velocity of $1.8 \mathrm{~cm} \mathrm{~s}^{-1}\left(Q=13.3 \mathrm{~cm}^{3} \mathrm{~s}^{-1}\right)$. The insets illustrate the flow contours. 20 levels were plotted with starting and outlet points at the inlet and exit of the reactor.

Fig. 5. Velocity magnitude profiles at different lengths: $x=0.3 \mathrm{~cm}, x=3 \mathrm{~cm}, x=8 \mathrm{~cm}$ and $x=15 \mathrm{~cm}$; at depth of $0.1 \mathrm{~cm}$ (in each cell). Mean linear flow velocity of $1.8 \mathrm{~cm} \mathrm{~s}^{-1}$ $\left(Q=13.3 \mathrm{~cm} 3 \mathrm{~s}^{-1}\right)$.

Fig. 6. Comparison between theoretical (_- ) and experimental (-----) RTD curves at different inflow volumetric rates showed in the figure.

Fig. 7. Comparisons of the theoretical concentration distribution in the three cells at different times in the $x-y$ plane at a depth of $0.1 \mathrm{~cm}$ (in each cell). Inflow volumetric rate of 
$13.3 \mathrm{~cm}^{3} \mathrm{~s}^{-1}(\tau=9.8 \mathrm{~s})$.

Fig. 8. Streamlines in the FM01-LC reactor at an inflow volumetric rate of $13.3 \mathrm{~cm}^{3} \mathrm{~s}^{-1}$. (a) Lateral view in the $x-z$ plane, and (b) back view of the third cell in the $x-y$ plane. 300 streamlines was plotted, with starting and outlet points at the inlet and exit of the filterpress reactor.

Fig. 9. Logarithmic plot of the pressure drop against the Reynolds number for the FM01LC electrochemical reactor containing a stack of three empty cells ( $\square$ ) (this work); this is compared with that obtained in an empty single cell $(\Delta)$ [27], and a filled single cell with PTFE turbulence promoter type $\mathrm{D}(\diamond)[27]$. 


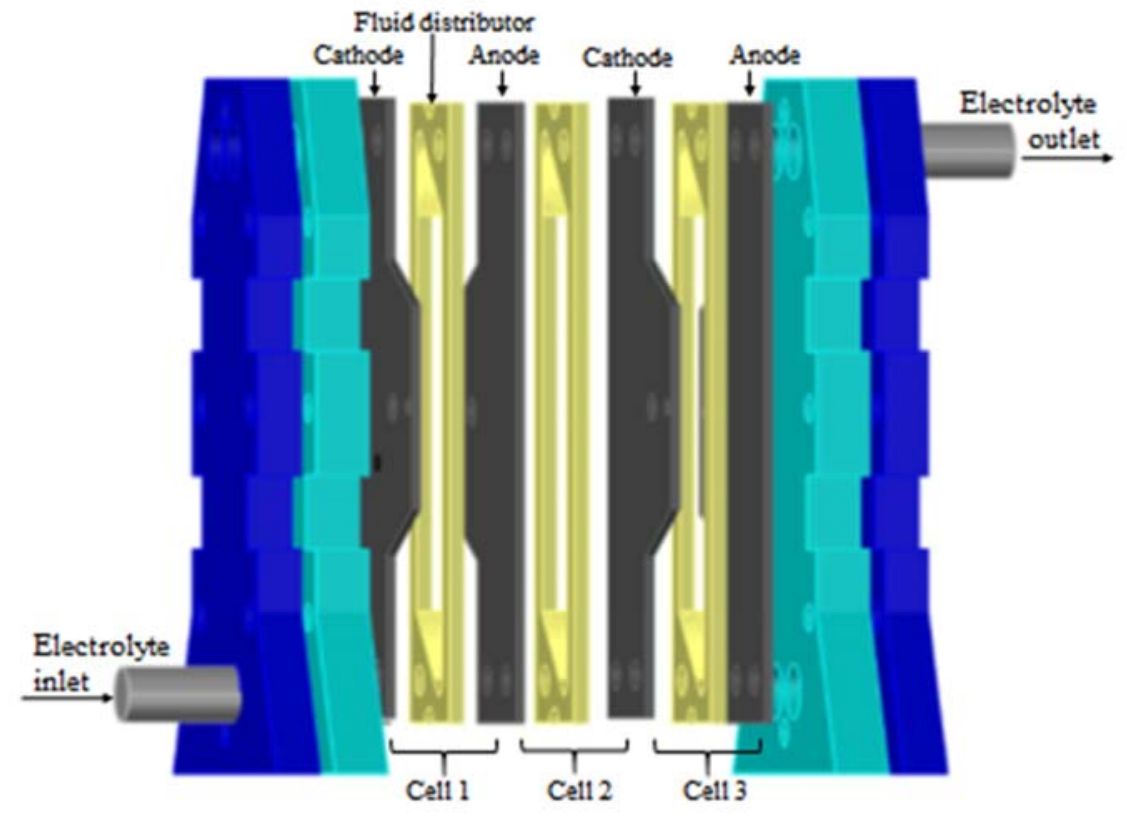

Figure 1
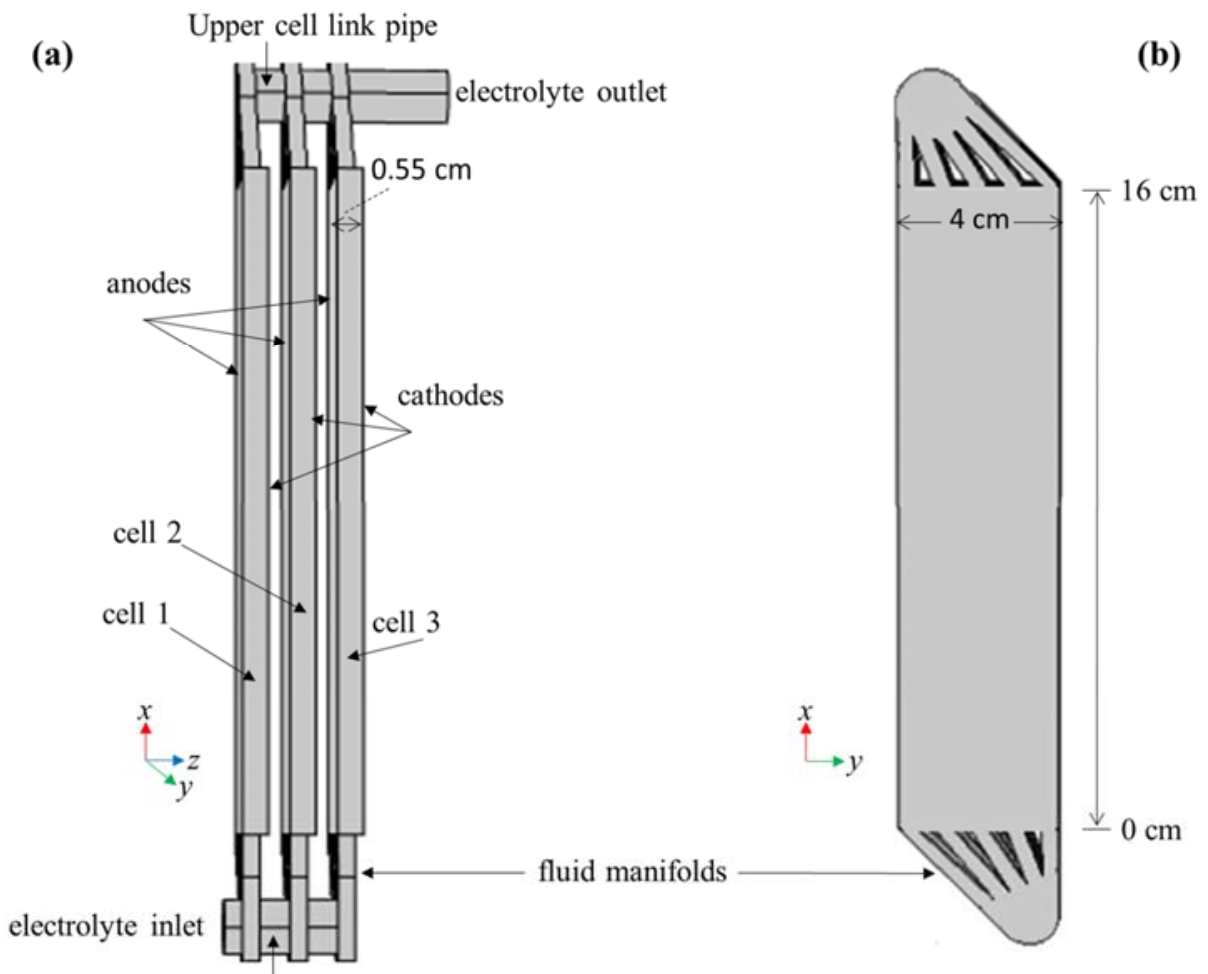

Bottom cell link pipe

Figure 2 


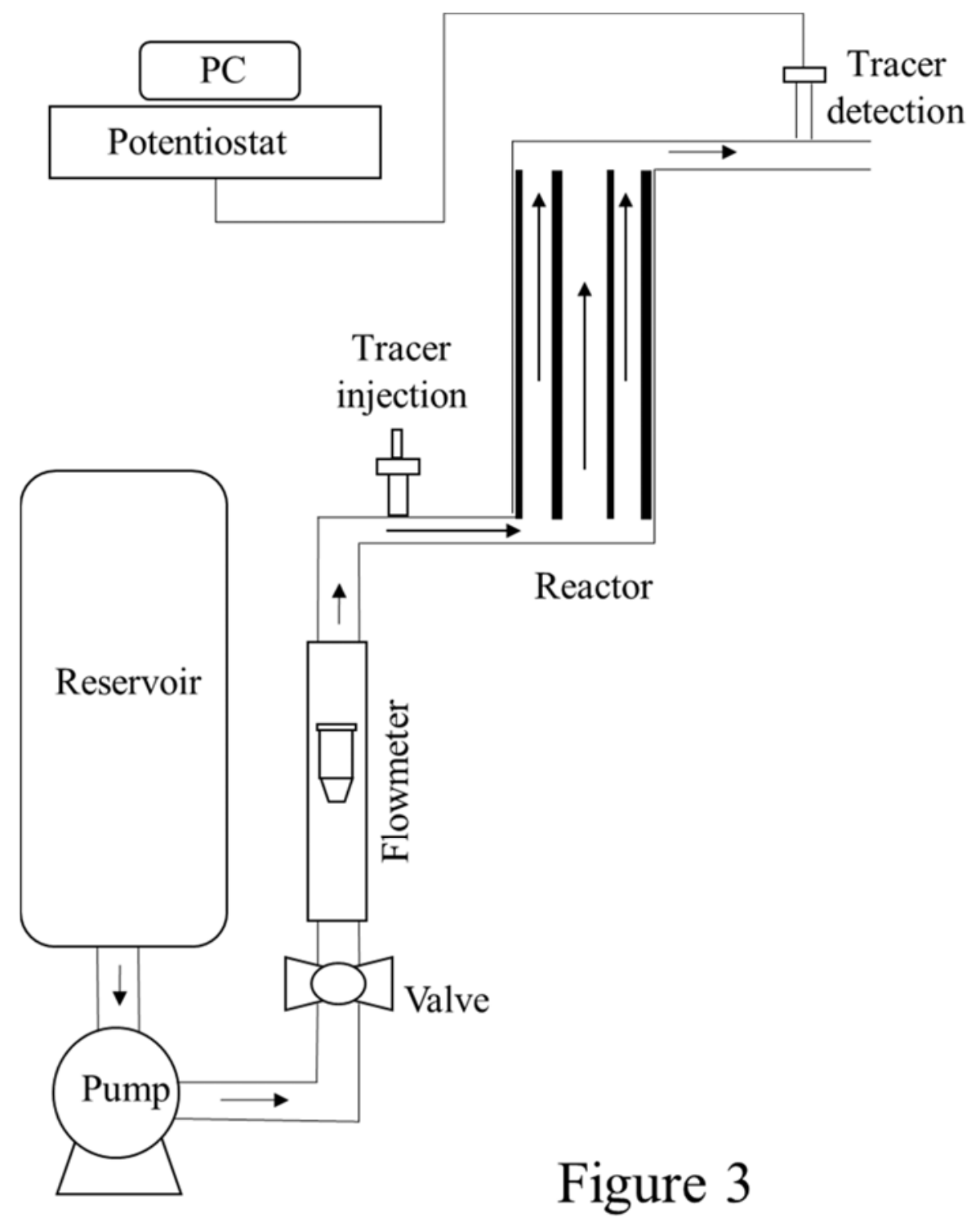




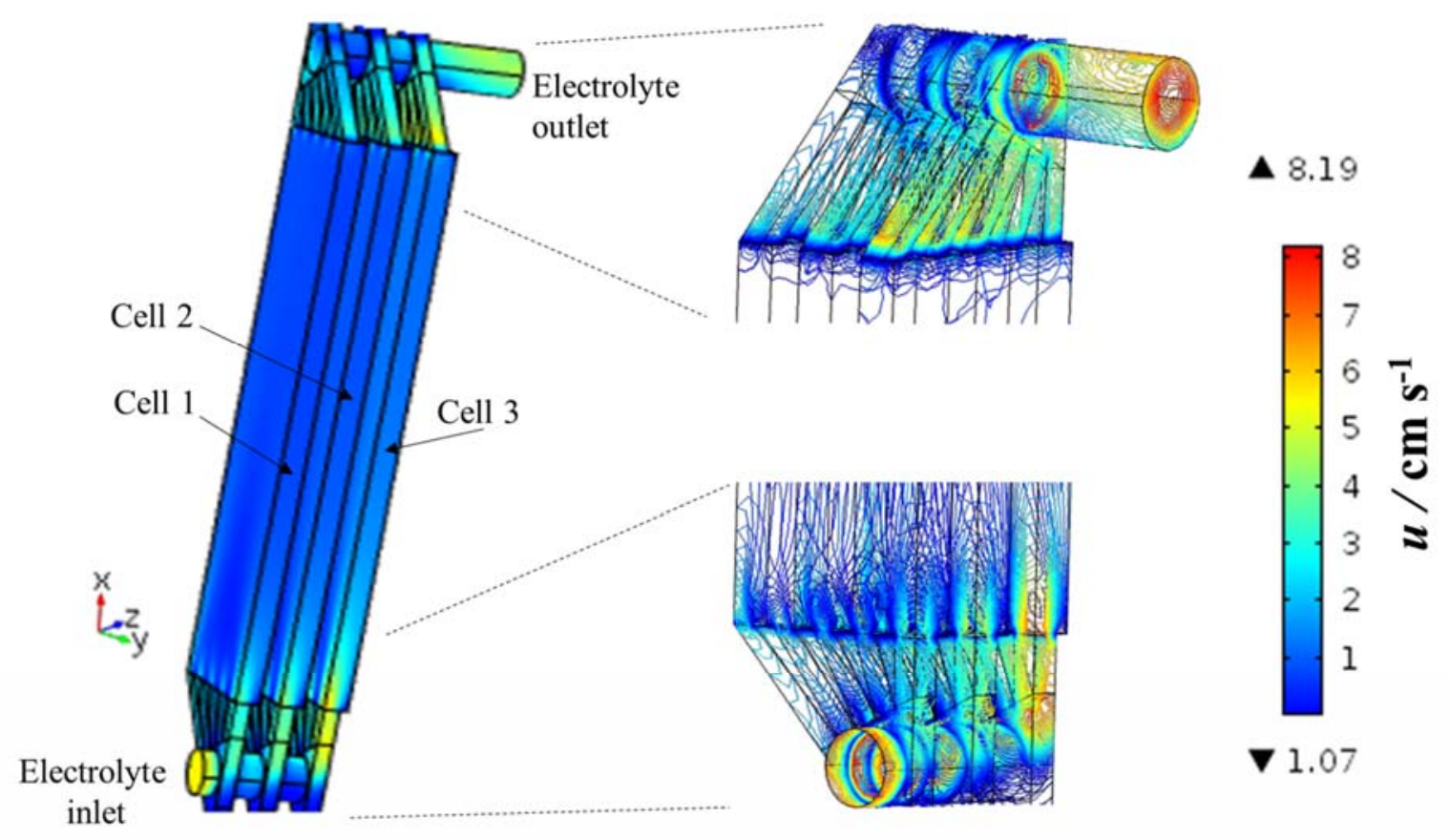

Figure 4
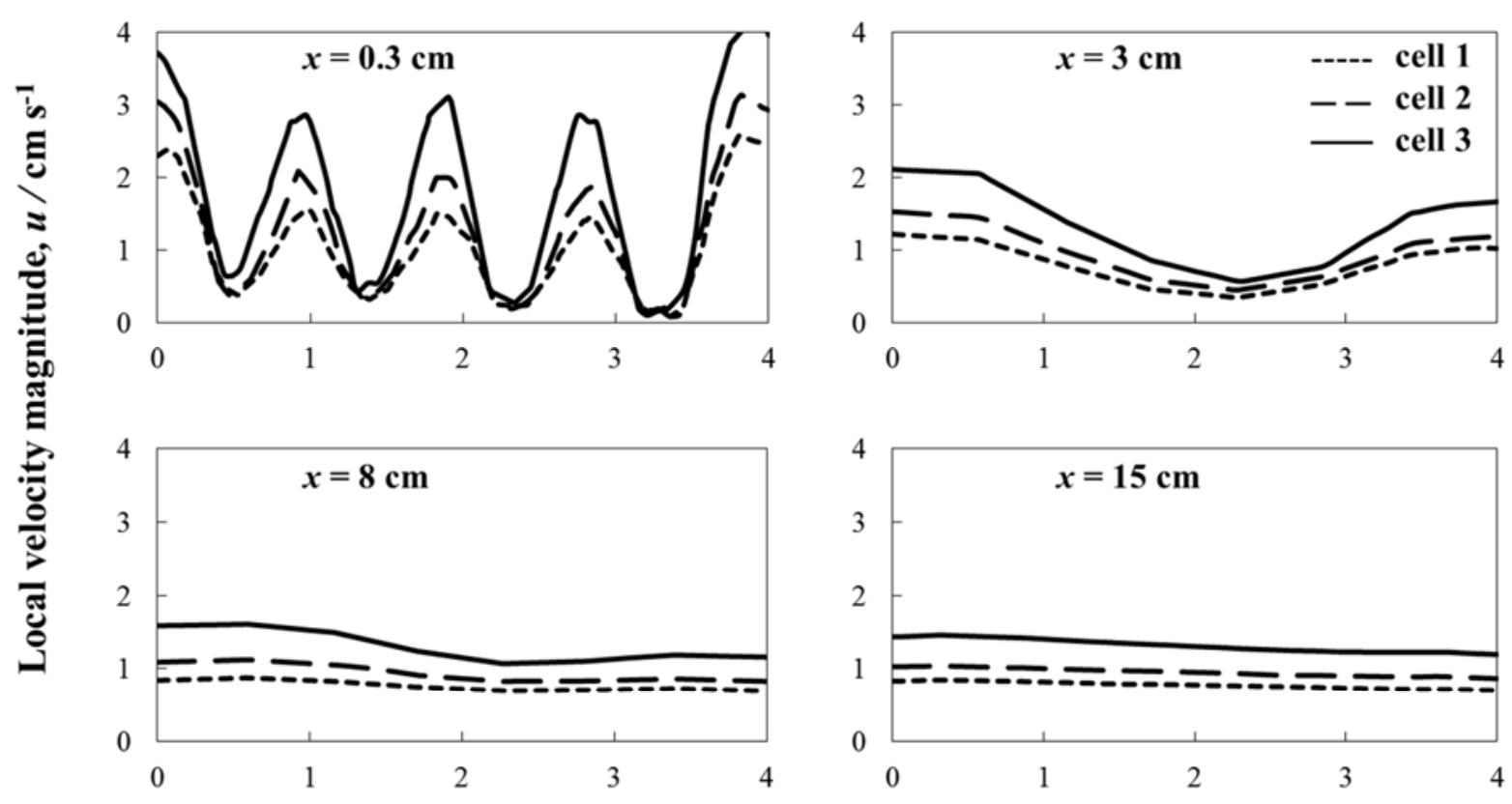

Channel width $/ \mathrm{cm}$

Figure 5 

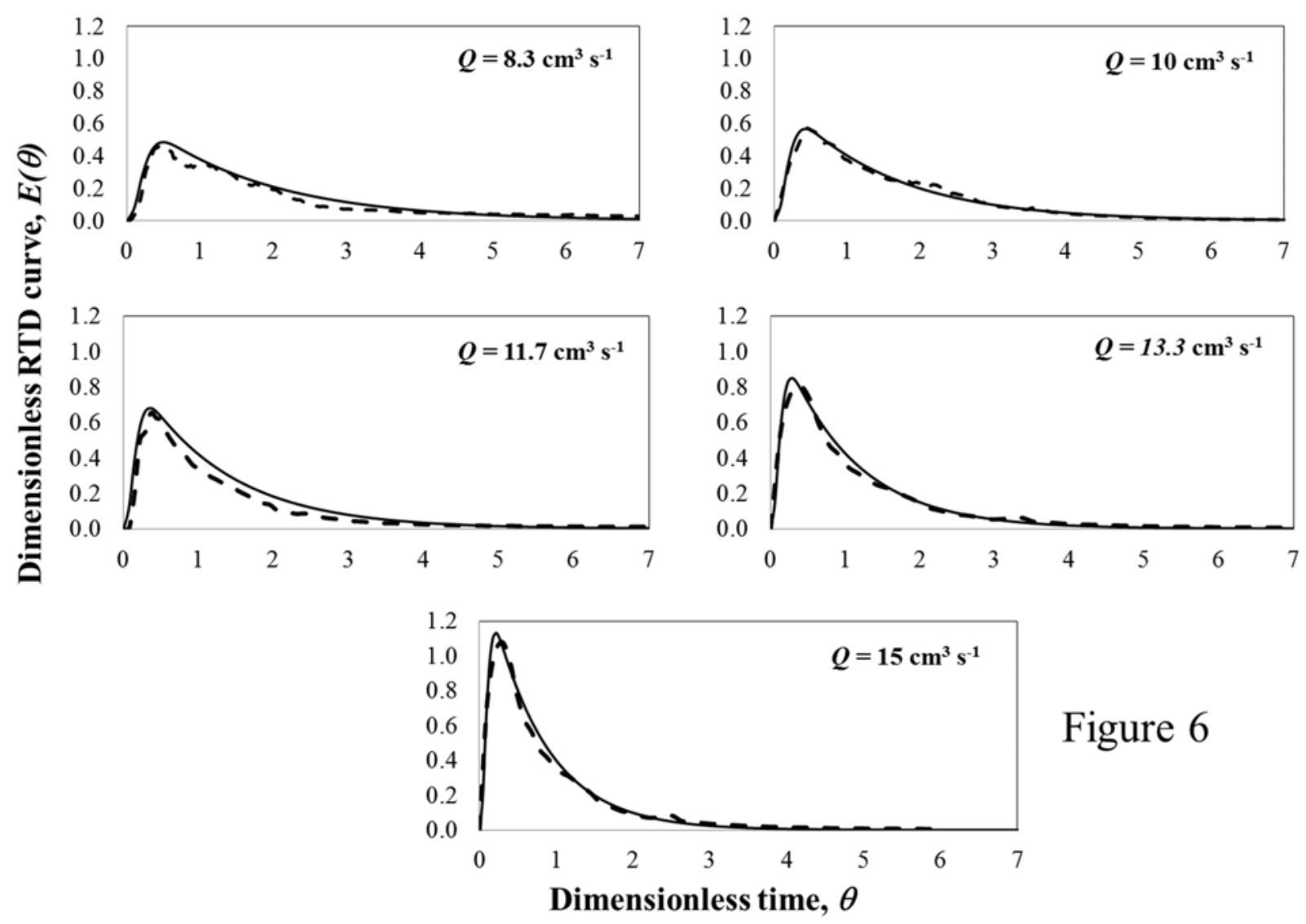

Figure 6 


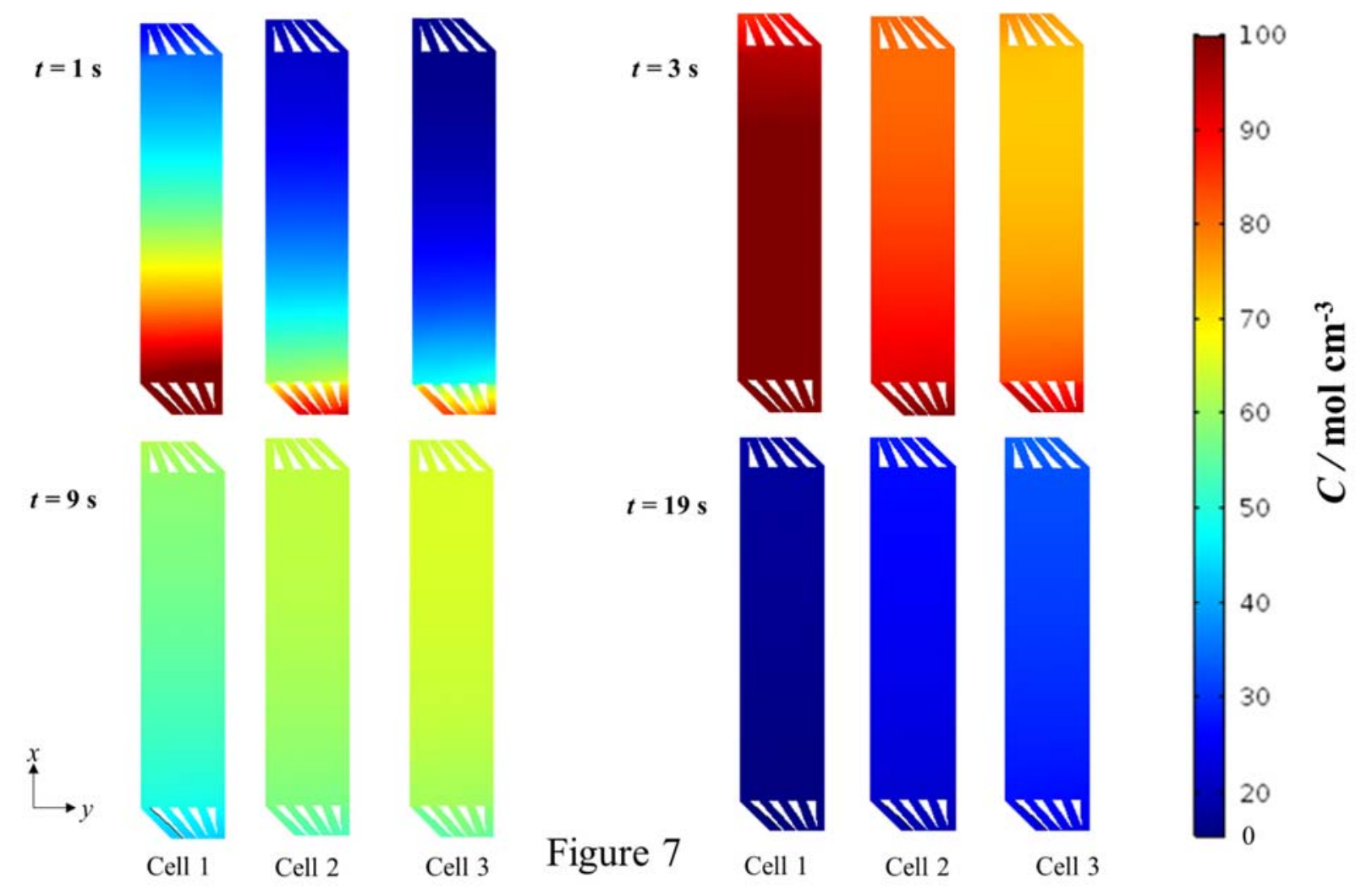




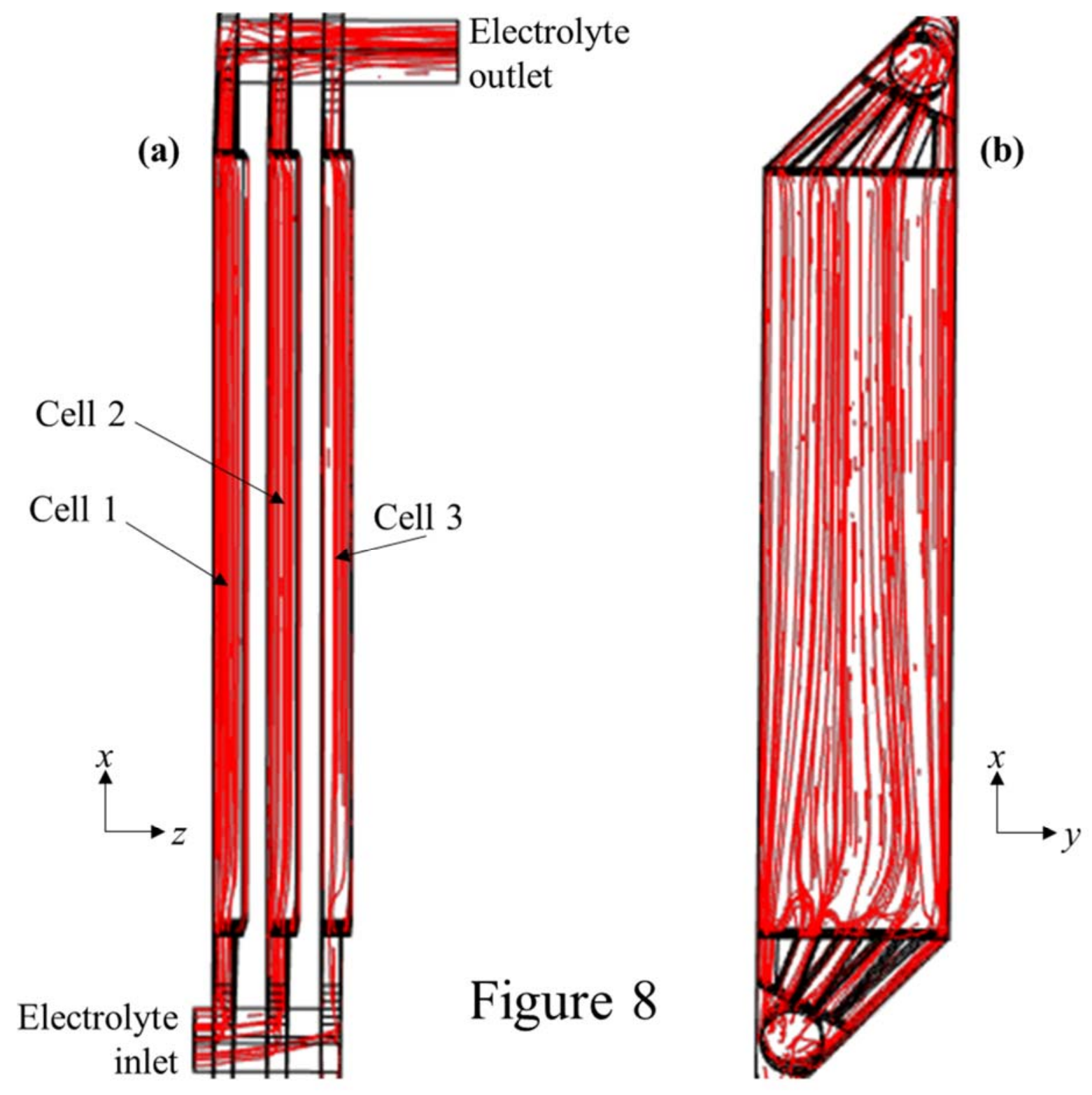




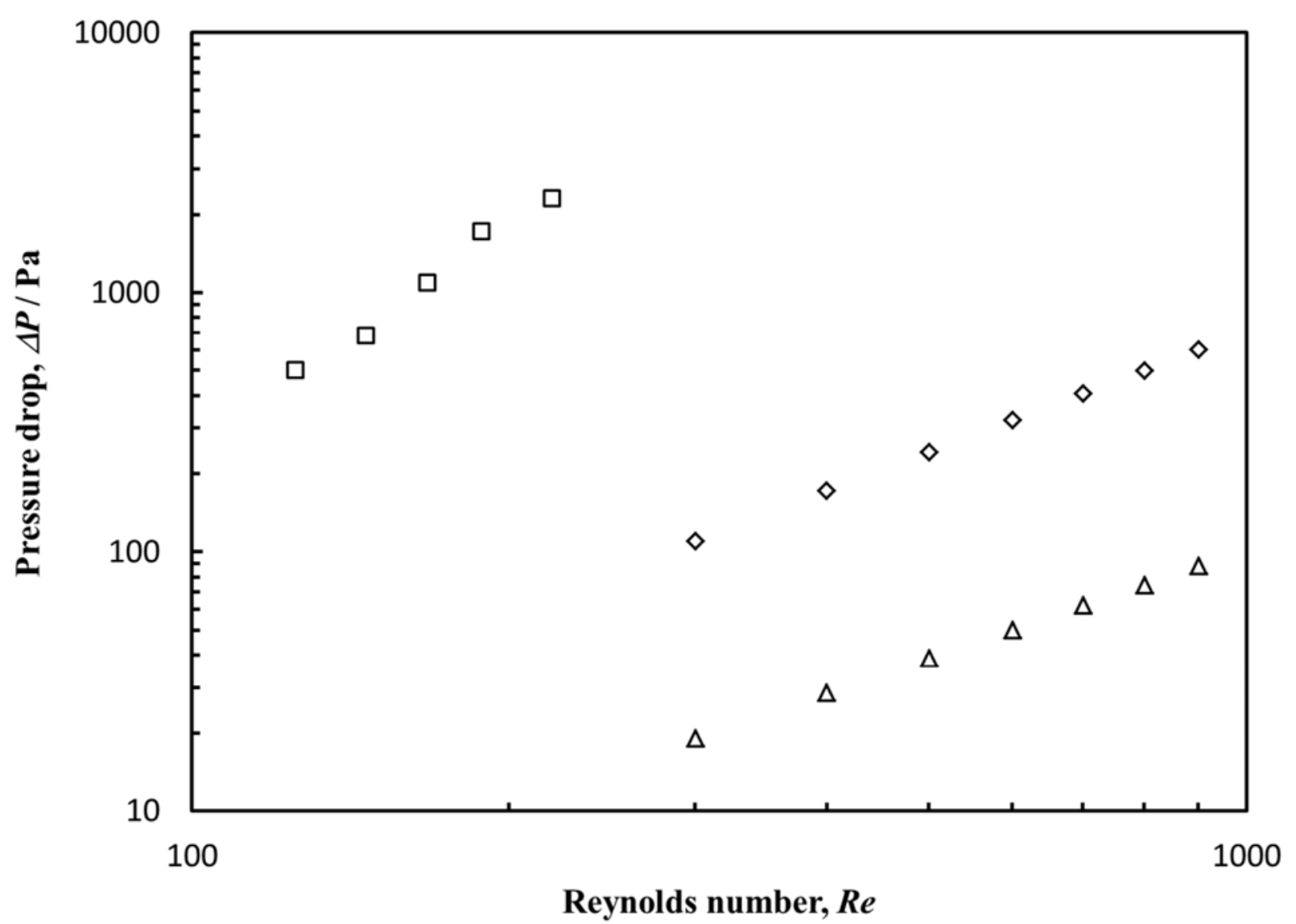

Figure 9 